\title{
sciendo
}

\section{The Effects of the Movement Tempo on the One-Repetition Maximum Bench Press Results}

\author{
by \\ Michal Wilk ${ }^{1}$ Artur Golas ${ }^{1}$, Piotr Zmijewski ${ }^{2}$, Michal Krzysztofik ${ }^{1}$, \\ Aleksandra Filip ${ }^{1}$, Juan Del Coso ${ }^{3}$, James J. Tufano ${ }^{4}$
}

\begin{abstract}
Different tempos of movement can be used during resistance training, but programming them is often a trialand-error practice, as changing the speed at which the exercise is performed does not always correspond with the tempo at which the 1-repetition-maximum occurred. Therefore, the aim of this study was to determine the effect of different movement tempos during the bench press (BP) exercise on the one-repetition maximum (1RM) load. Ninety men (age = $25.8 \pm 5.3$ years, body mass $=80.2 \pm 14.9 \mathrm{~kg}$ ), with a minimum one year of resistance training experience took part in the study. Using a randomized crossover design, each participant completed the BP 1RM test with five different movement tempos: V/0/V/0, 2/0/V/0,5/0/V/0, 8/0/V/0 and 10/0/V/O. Repeated measures ANOVA compared the differences between the 1RM at each tempo. The 1RM load was significantly greater during $V / 0 / V / 0$ and 2/0/V/0 compared to $5 / 0 / V / 0,8 / 0 / V / 0$, and $10 / 0 / V / 0(p<0.01)$. Furthermore, the 1RM load was significantly greater during $5 / 0 / V / 0$ compared to 8/0/V/O and 10/0/V/0 $(p<0.01)$, but there were no differences between either V/O/V/0 and 2/0/V/0 $(p=0.92)$ or between $8 / 0 / V / 0$ and $10 / 0 / V / 0(p=0.08)$. Therefore, different movement tempos used during training should be accompanied by their own tempo-specific 1RM testing, as slower eccentric phases significantly decrease maximal concentric performance. Furthermore, 1RM test procedures should include information about the movement tempo used during the test protocol. In addition, the standardization of the tempo should be taken into account in investigations that use the 1 RM test to assess the effects of any treatment on maximal muscle strength.
\end{abstract}

Key words: eccentric movements, duration of repetition, maximal strength, external load, resistance training.

\section{Introduction}

Exercise intensity is one of the key variables in training and has a significant effect on training adaptations. In resistance training, exercise intensity is usually prescribed from a certain repetition maximum (RM), which can be represented by a range (10-12RM) or as a percentage of one-repetition maximum (1RM; e.g. $85 \%$ of $1 \mathrm{RM}$ ) (Shimano et al., 2006). The 1RM is defined as the maximal load that the athlete can lift once with proper technique and it is highly specific to the exercise at which the $1 R M$ is measured. 1RM testing is a very common evaluation and training tool in sport science because it is relatively simple and does not require expensive laboratory equipment (Kraemer et al., 2006). In addition, the 1 RM is considered the gold standard for assessing muscle strength under non-laboratory conditions (Levinger et al., 2009). Although the 1RM is commonly used in resistance exercise methodology, previous testing procedures to assess $1 \mathrm{RM}$ in a standardized manner do not provide specific guidelines related to the movement tempo or the duration of particular phases of the exercise (eccentricconcentric) (Mike et al., 2017; Seo et al., 2012; Wilk

\footnotetext{
1 - Institute of Sport Sciences, Jerzy Kukuczka Academy of Physical Education in Katowice, Poland.

2 - Jozef Pilsudski University of Physical Education in Warsaw, Warsaw, Poland.

3 - Centre for Sport Studies, Rey Juan Carlos University, Fuenlabrada, Spain.

4 - Faculty of Physical Education and Sport, Charles University, Prague, Czech Republic.
} 
et al., 2018a), which can be problematic in terms of using different tempos in the periodization process. Furthermore, some previous studies showed that maximal strength and power output production depend on training status (Miller et al., 2018) and motivation (Maszczyk et al., 2019, 2020).

Movement tempo is often defined as the pace, cadence or lifting speed of a resistance exercise (Wilk et al., 2018a, 2019). Thus, tempo is usually described using a sequence of digits (e.g. $2 / 0 / 3 / 0)$, where each digit determines the duration, in seconds, of a particular phase of the movement (eccentric / isometric / concentric / isometric) while " $\mathrm{V}$ " is used to indicate that the phase should be performed at a volitional tempo and " $X$ " indicates a phase that is done explosively or as fast as possible. In this paper we unified the description of the movement tempo, using the following terms: eccentric / isometric / concentric / isometric and four-digit combinations.

In practice, strength training programs can make use of various movement tempos, but prescribing exercises with different tempos is more of a "trial and error" practice than one that is evidence-based. In fact, only two studies have examined the impact of different movement tempos on the maximal load lifted during 1RM testing (Headley et al., 2011; Wilk et al., 2020). Headley et al. (2011) showed that the bench press (BP) $1 \mathrm{RM}$ was significantly greater $(3.7 \%)$ during a faster tempo (2/0/2/0) compared to a slower one $(4 / 0 / 2 / 0)$. Although valuable, this information does not account for the fact that most concentric phases of RM testing are performed either as quickly or explosively as possible or with voluntary duration. Therefore, Wilk et al. (2020) investigated the effects of different eccentric (ECC) phases paired with non-restrictive, explosive concentric $(\mathrm{CON})$ phases. They showed that the 1RM load was significantly greater during the BP performed with a $2 / 0 / \mathrm{X} / 0$ tempo compared to $4 / 0 / \mathrm{X} / 0(44.6 \pm 3.5$ vs. $41.8 \pm 3.6$, respectively) and $6 / 0 / \mathrm{X} / 0$ tempos (44.6 \pm 3.5 vs. $40.6 \pm 4.4$, respectively), with the $4 / 0 / \mathrm{X} / 01 \mathrm{RM}$ also being greater than the $6 / 0 / \mathrm{X} / 01 \mathrm{RM}(41.8 \pm 3.6 \mathrm{vs}$. $40.6 \pm 4.4$, respectively). These results suggest that a slower movement tempo, which means longer duration of the ECC phase of movement, decreased the maximal load lifted in the 1RM test. Given the widespread use of various tempos in practice, it would be valuable to determine how extending the ECC phase affects 1RM results, and ultimately load prescription. Considering the above, the periodization of different movement tempos and training loads can be more evidencebased rather than "trial and error" practice.

To the best of our knowledge, there are no data regarding the differences in 1RM performance between faster and slower tempos by manipulating the ECC phase of movement to be longer than $6 \mathrm{~s}$. Considering that ECC phases lasting 2 s (Headley et al., 2011; Wilk et al., 2020) and $4 \mathrm{~s}$ (Wilk et al., 2020) resulted in decreased $1 R M$ loads, it can be speculated that extending the ECC phase may further decrease the 1RM load compared to faster movement tempos. Therefore, the aim of this study was to determine the effects of different movement tempos (from volitional to $10 \mathrm{~s}$ in the ECC phase), during the bench press (BP) exercise on 1RM test results. Our initial hypothesis was that lengthening the ECC phase would result in smaller values of $1 \mathrm{RM}$.

\section{Methods}

\section{Experimental approach}

The experiment was performed following a randomized crossover design, where each participant acted as his own control. Each participant performed two familiarization sessions, one with 1RM testing, followed by five experimental sessions that were identical except for the movement tempo used. During each experimental session, participants completed a 1RM BP test with either a: V/0/V/0 volitional tempo (VOL), 2/0/V/0 fast tempo (FAS), 5/0/V/0 medium tempo (MED), 8/0/V/0 slow tempo (SLO) or extremely slow tempo 10/0/V/0 (ESLO). For example, $2 / 0 / \mathrm{V} / 0$ denotes a 2 s ECC phase, no pause during the transition phase, and a volitional movement tempo during the CON phase. The entire research procedure lasted 6 to 7 weeks, with experimental sessions performed 4 to 5 days apart. Participants were required to refrain from resistance training 72 hours prior to each experimental session, were familiarized with the exercise protocol and were informed about the benefits and risks of the experiment before expressing their written consent for participation in the study. Except for the standardizations set before each trial, participants maintained their training and diet routines to maintain a stable 
level of physical fitness during the whole experiment. All testing was performed in the Strength and Power Laboratory at the Jerzy Kukuczka Academy of Physical Education in Katowice.

\section{Participants}

Ninety (90) healthy men (age $=25.8 \pm 5.3$ years, body mass $=80.2 \pm 14.9 \mathrm{~kg}$ ), with a minimum one year of strength training experience $(3.9 \pm 4.2$ years) took part in the research. In order to exclude the effect of motor learning, at least 3 weeks before the study, all participants practiced the BP with different movement tempos. Participants were allowed to withdraw from the experiment at any moment and were free of any pathologies or injuries. The study was carried out in accordance with the recommendations of the ethical standards of the Declaration of Helsinki, 1983. The protocol was approved by the Bioethics Committee for Scientific Research, at the Academy of Physical Education in Katowice, Poland (10/2018). Participants were instructed to avoid any dietary supplements or stimulants for the duration of the study.

\section{Procedures}

Familiarization session

Three weeks before the onset of the experimental sessions, participants performed two familiarization sessions separated by $4-5$ days. During the familiarization sessions, participants performed 5 sets of 2 repetitions of the BP with approximately $70 \%$ of their selfreported 1RM. To restrict any possible learning effects, every set was performed with a different movement tempo described above. One week before the first experimental session, initial 1RM testing was performed. For 1 RM testing, participants arrived at the laboratory at the same time of day as the upcoming experimental sessions and cycled on an ergometer for 5 minutes, followed by a general upper body warmup of 10 body weight pull ups and 15 body weight push-ups. Next, athletes performed 15, 10, and $5 \mathrm{BP}$ repetitions using $20 \%, 50 \%$, and $70 \%$ of their estimated 1RM, respectively. The first testing load was set to an estimated $80 \% 1 \mathrm{RM}$, and was increased by 2.5 to $5 \mathrm{~kg}$ and the process was repeated until failure. During the 1RM test, participants executed one repetition with a tempo of $\mathrm{V} / 0 / \mathrm{V} / 0$ and $5 \mathrm{~min}$ rest intervals between successful trials. Hand placement on the barbell was set at $150 \%$ of the individual bi-acromial distance. The positioning of the hands was recorded to ensure consistent hand placement during all experimental sessions.

Experimental sessions

The general and specific warm-ups for the experimental sessions were identical to the ones used for the familiarization session. The specific BP warm-up was performed with a volitional tempo. Starting from a load of approximately $80 \% 1 \mathrm{RM}$, all the subsequent sets and attempts to obtain $1 \mathrm{RM}$ were performed with the randomly selected tempo for that day. The movement tempo in the ECC phase was guided by a metronome. The CON phase of the BP movement was performed at the volitional tempo (V). In each subsequent attempt, the load was increased by 2.5 to $10 \mathrm{~kg}$, and the protocol was repeated until the load that represented the $1 \mathrm{RM}$ was reached. The rest interval between particular trials was $5 \mathrm{~min}$. All repetitions were performed without bouncing the barbell off the chest and without raising the lower back off the bench. All familiarization and experimental sessions were recorded by means of a Sony camera (FDR191). The correctness of particular repetitions performed at a specific tempo of movement was checked immediately after the completion of each repetition (from recorded data). In the event of incorrect performance according to the tempo of movement, the attempt was repeated.

\section{Statistical analysis}

All statistical analyses were performed using Statistica 9.1 and Microsoft Excel, and the results were presented as means with standard deviations. The Shapiro-Wilk, Levene and Mauchly's tests were used in order to verify the normality, homogeneity and sphericity of the sample data variances, respectively. A one-way ANOVA with repeated measures was used to compare the differences between the considered variables. In the event of a significant main effect, post hoc comparisons were conducted using the Tukey's post hoc test. The statistical significance for the differences between tempos was set at $p<$ 0.05 . Percentage changes and $95 \%$ confidence intervals were also calculated. Relative differences (\%) between the analyzed variables and Effect Sizes (Cohen's $d$ ) were determined. The ES was interpreted as large for $d>0.8$, moderate for $d$ between 0.8 and 0.5 , and small for $d<0.5$ (Cohen, 2013). 


\section{Results}

The ANOVA revealed a statistically significant main effect of the movement tempo on 1 RM values $(p<0.01$; Table 1$)$. The post-hoc tests showed that the maximal load in the 1RM test was significantly greater during the $2 / 0 / \mathrm{V} / 0$, as well as during the $\mathrm{V} / 0 / \mathrm{V} / 0$ tempo when compared to the slower tempo of $5 / 0 / \mathrm{V} / 0,8 / 0 / \mathrm{V} / 0$ and 10/0/V/0 $(p<0.01$; Table 2). Furthermore, the maximal load in the 1RM test was significantly greater during the 5/0/V/0 tempo when compared to the slow 8/0/V/0 and extremely slow 10/0/V/0 movement tempo $(p<0.01$; Table 2$)$. There were no significant differences in the maximal load lifted during the $1 \mathrm{RM}$ test between the volitional $(\mathrm{V} / 0 / \mathrm{V} / 0)$ and fast $(2 / 0 / \mathrm{V} / 0)$ tempo $(p=0.92)$, as well as between the slow $(8 / 0 / \mathrm{V} / 0)$ and extremely slow $(10 / 0 / \mathrm{V} / 0)$ movement tempo $(p=0.08$; Table 2).

Table. 1

One-repetition maximum (1RM) performance with different movement tempos Movement tempo during the 1RM test

\begin{tabular}{ccccc}
\hline $\mathrm{V} / 0 / \mathrm{V} / 0$ & $2 / 0 / \mathrm{V} / 0$ & $5 / 0 / \mathrm{V} / 0$ & $8 / 0 / \mathrm{V} / 0$ & $10 / \mathrm{V} / 0 / 0$ \\
$(95 \% \mathrm{CI})$ & $(95 \% \mathrm{CI})$ & $(95 \% \mathrm{CI})$ & $(95 \% \mathrm{CI})$ & $(95 \% \mathrm{CI})$
\end{tabular}

\begin{tabular}{lcccccc}
$1 \mathrm{RM}$ & & & & & \\
$(\mathrm{kg})$ & $121.4 \pm 49.6$ & $120.9 \pm 49.2$ & $113.8 \pm 47.1$ & $107.1 \pm 44.5$ & $105.9 \pm 43.0$ & $0.01^{*}$ \\
& $(111.0$ to & $(110.6$ to & $(103.9$ to & $(97.8$ to & $(96.8$ to & \\
& $131.7)$ & $131.3)$ & $123.6)$ & $116.5)$ & $114.9)$ & \\
\hline
\end{tabular}

All data are presented as mean \pm standard deviation with $95 \%$ confidence intervals; ${ }^{*}$ statistically significant differences at $p<0.05$

Table. 2

A summary of tempo comparisons.

\begin{tabular}{cccccc}
\hline \multirow{2}{*}{ Movement tempo } & $p$ & $\begin{array}{c}\text { Effect size } \\
\text { (Cohen } d)\end{array}$ & $\begin{array}{c}\text { Relative effect } \\
(\%)\end{array}$ \\
\cline { 2 - 6 } & $\mathrm{V} / 0 / \mathrm{V} / 0$ & $2 / 0 / \mathrm{V} / 0$ & 0.92 & 0.01 & 0.4 \\
$\mathrm{~V} / 0 / \mathrm{V} / 0$ & $5 / 0 / \mathrm{V} / 0$ & $0.01^{*}$ & 0.16 & 6.3 \\
& $\mathrm{~V} / 0 / \mathrm{V} / 0$ & $8 / 0 / \mathrm{V} / 0$ & $0.01^{*}$ & 0.30 & 11.8 \\
& $\mathrm{~V} / 0 / \mathrm{V} / 0$ & $10 / 0 / \mathrm{V} / 0$ & $0.01^{*}$ & 0.33 & 12.8 \\
$1 \mathrm{RM}(\mathrm{kg})$ & 2/0/V/0 & $5 / 0 / \mathrm{V} / 0$ & $0.01^{*}$ & 0.15 & 5.8 \\
& 2/0/V/0 & $8 / 0 / \mathrm{V} / 0$ & $0.01^{*}$ & 0.29 & 11.4 \\
& 2/0/V/0 & $10 / 0 / \mathrm{V} / 0$ & $0.01^{*}$ & 0.33 & 12.4 \\
& $5 / 0 / \mathrm{V} / 0$ & $8 / 0 / \mathrm{V} / 0$ & $0.01^{*}$ & 0.15 & 5.9 \\
& $5 / 0 / \mathrm{V} / 0$ & $10 / 0 / \mathrm{V} / 0$ & $0.01^{*}$ & 0.18 & 6.9 \\
& $8 / 0 / \mathrm{V} / 0$ & $10 / 0 / \mathrm{V} / 0$ & 0.08 & 0.03 & 1.1 \\
\hline
\end{tabular}

${ }^{*}$ statistically significant differences at $p<0.05$ 
Table 3

Hypothetical relationships between different training volume indices including total time under tension (TUT) according to different 1-repetiton maximum (1RM) scores for different

\begin{tabular}{lccccc}
\multicolumn{5}{c}{ movement tempos. } \\
\hline Tempo & $2 / 0 / 1 / 0$ & $5 / 0 / 1 / 0$ & $8 / 0 / 1 / 0$ & $10 / 0 / 1 / 0$ & $10 / 0 / 1 / 0$ \\
\hline 1RM & 150 & 140 & 130 & 125 & 125 \\
$75 \% 1 \mathrm{RM}$ & $113 \mathrm{~kg}$ & $105 \mathrm{~kg}$ & $98 \mathrm{~kg}$ & $94 \mathrm{~kg}$ & $94 \mathrm{~kg}$ \\
Number of repetitions & $15 \mathrm{reps}$ & $15 \mathrm{reps}$ & $15 \mathrm{reps}$ & $15 \mathrm{reps}$ & $5 \mathrm{reps}$ \\
Load*Reps & $1.695 \mathrm{~kg}$ & $1.575 \mathrm{~kg}$ & $1.470 \mathrm{~kg}$ & $1.410 \mathrm{~kg}$ & $470 \mathrm{~kg}$ \\
Load*Reps*TUT $^{*}$ & $5.085 \mathrm{~kg}^{*} \mathrm{~s}$ & $9.450 \mathrm{~kg}^{*} \mathrm{~s}$ & $13.230 \mathrm{~kg}^{*} \mathrm{~s}$ & $15.510 \mathrm{~kg}^{*} \mathrm{~s}$ & $5.170 \mathrm{~kg}^{*} \mathrm{~s}$ \\
\hline
\end{tabular}

These examples assume that the tempo of movement remains constant and does not account for fatigue during the concentric phase, which may result in slightly different values. In the final column the 10/0/1/0 tempo results are similar in Load ${ }^{*}$ Reps*TUT to those of the 2/0/1/0 tempo, despite a smaller load and fewer repetitions.

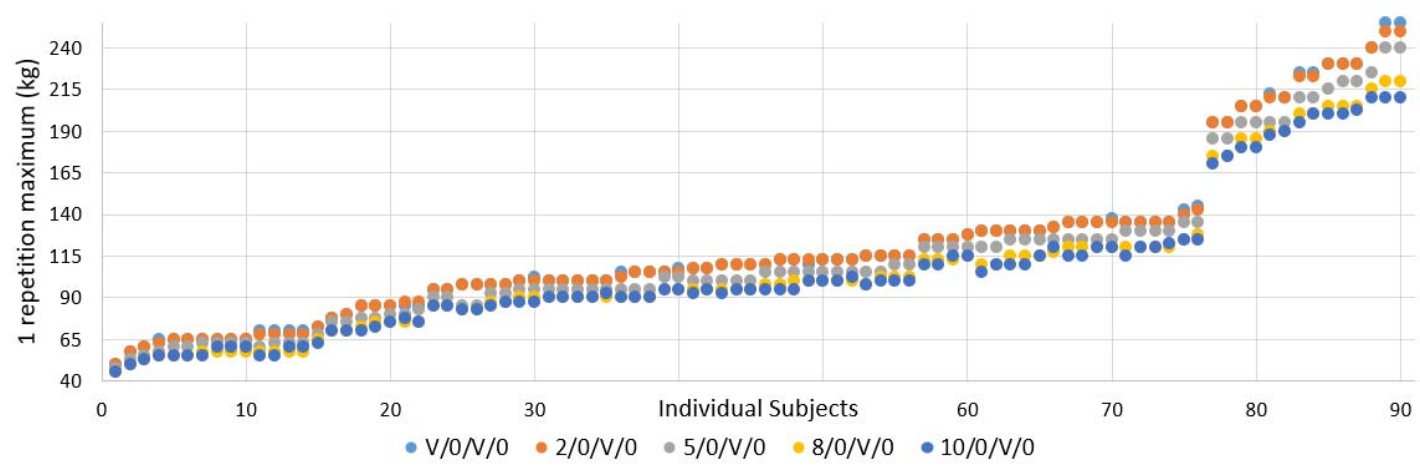

Figure 1

The absolute 1 repetition maximum for 5 different movement tempos.

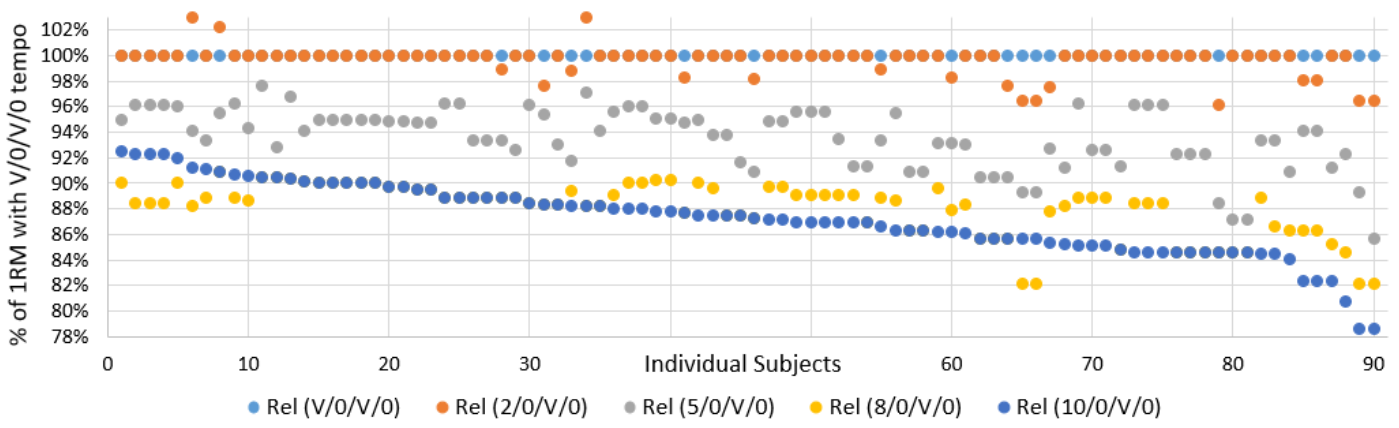

Figure 2

The 1 repetition maximum relative to the V/0/V/0 tempo. 


\section{Discussion}

The main finding of this study was that increasing the duration of the ECC phase of the bench press 1RM testing significantly decreased the maximal 1RM load. The data in Table 1 and 2 suggest that the movement tempo with volitional tempo or with duration up to $2 \mathrm{~s}$ during the ECC phase may be optimal for reaching maximal results in the bench press exercise, which is preferable for athletes who want to maximize their 1RM (e.g. powerlifters). However, for those who do not need to maximize their $1 \mathrm{RM}$, this study still provides valuable information that can be used for designing resistance training programs with variable movement tempos.

Overall, the results of our study indicate that the slower movement tempo during the bench press exercise, the lower the load lifted in a 1RM test, confirming our initial hypothesis (Table 1; Figure 1). The results are also consistent with Headley et al. (2011) and Wilk et al. (2020) who showed that slower movement tempos significantly decreased the maximal load lifted in a BP 1RM test. In the study of Headley et al. (2011), their participants were able to lift a 3.7\% greater maximal load during the $1 \mathrm{RM}$ test with a $2 / 0 / 2 / 0$ compared to a 4/0/2/0 tempo. Similarly, participants in the Wilk et al.'s (2020) study were able to lift $6.2 \%$ and $9.1 \%$ greater maximal loads during the $1 \mathrm{RM}$ test with a 2/0/2/0 tempo compared to $4 / 0 / \mathrm{X} / 0$ and $6 / 0 / \mathrm{X} / 0$ tempos, respectively. However, the present study is the first one considering slow and extremely slow movement tempos with 8- and 10-s duration of the ECC phase of movement. Our study shows that an additional extension of the ECC phase, further decreases the 1RM load. The differences between the FAS $(2 / 0 / \mathrm{V} / 0)$ and ESLO $(10 / 0 / \mathrm{V} / 0)$ tempo reached more than $10 \%$ of the load reached in the 1RM test (individual responses in Figure 2), which is significant not only in statistical terms, but also in sports training and scientific research. The absolute difference in $\mathrm{kg}$ between the two extreme movement tempos used in this investigation (i.e., FAS and ESLO) equaled to an average of $15.5 \mathrm{~kg}$. However, it should be noted that in the strongest participants, differences between the FAS and ESLO tempo amounted to more than $25 \mathrm{~kg}$ (see Table 3 for a practical example of how this may affect training volume indices).
Despite the fact that our study confirmed that increasing the duration of the ECC phase decreased the 1RM load, there were no differences between the VOL and FAS tempos or between the SLO and ESLO movement tempos. The lack of differences between the VOL and FAS tempos can be explained by the lack of significant differences in the duration of the ECC phases of movement between the two test conditions. An analysis of the video recordings obtained during each protocol indicated that the duration of the ECC phase in the VOL tempo was similar to the duration of the FAS tempo (approximately $2 \mathrm{~s}$ ) which explains the lack of significant differences. When comparing the SLO and ESLO tempo, the differences in 1RM were not significant, despite no significant differences in 1RM, there was a trend for decreased results with increased duration of the ECC phase of movement (107.1 \pm $44.5 ; 105.9 \pm 43.0$ for SLO, ESLO, respectively). The relative differences (\%) in 1RM test results (Figure 2) between the SLO and the ESLO tempo were $1.1 \%$, and although such changes in results of the $1 \mathrm{RM}$ test may be considered small in statistical terms, this difference may be of great significance in training of elite athletes as well as in scientific research.

Similar to the Wilk et al.'s (2020) study, the main factor influencing 1RM performance was the duration of the ECC phase of movement. The duration of effort in this phase of the movement equaled 2, 5, 8 and $10 \mathrm{~s}$ for the FAS, MED, SLO and ESLO tempo, respectively. In this respect, the longer duration of effort in the slower trials likely implied greater muscle exhaustion and consequently premature exercise fatigue, which contributed to the decreases in the load lifted in the 1RM test (Wilk et al., 2020). The duration of effort during a single repetition as well as during all the repetitions in a set is referred to as the time under tension (TUT; (Wilk et al., 2018a)). Time under tension is an indicator of the effort performed, which includes the sum of the CON, ECC, and isometric components of a repetition (Schoenfeld et al., 2015). Therefore, TUT can be an indicator of exercise volume regardless of the number of repetitions performed, as indicated in Table 3. Previous studies showed that training with a slower movement tempo and longer TUT results in greater increases in blood lactate and testosterone concentration, compared to a faster 
tempo and shorter TUT (Goto et al., 2009; Martins-Costa et al., 2016; Mazzetti et al., 2007; Wilk et al., 2018b). Thus, extending the duration of a single repetition through a slower movement tempo is a significant factor increasing metabolic stress to resistance exercise, regardless of the number of performed repetitions. Thus, the longer TUT during the SLO and ESLO tempo compared to the FAS tempo indicated a higher exercise volume in these latter tempos. This leads to the conclusion that decreases in the result of 1RM testing during a slower movement tempo may be related to longer TUT.

Higher results of the 1RM performance during the faster movement tempos can also be related to a more effective use of elastic energy generated during the faster transition from the ECC to the CON phase of the movement (Cronin et al., 2001, 2004; Newton et al., 1997). Wilk et al. (2019) registered a decrease in maximal CON bar velocity when a slower ECC contraction was used, which was partially linked to less efficient utilization of the stretch-shortening cycle. A slower movement tempo in the ECC phase can cause a reduction in the efficiency of the stretchshortening cycle, which may partly explain the lower 1RM result.

The impact of the movement tempo on $1 \mathrm{RM}$ test results is important not only for assessing muscle strength, but also may be of importance in assessing the effect of different movement on adaptive changes following resistance training. Most of scientific research that described the impact of different movement tempos on acute and chronic adaptive changes following resistance training is based on the results of the 1RM test that was only performed with a volitional tempo of movement (Mike et al., 2017; Wilk et al., 2018a, 2019). On the basis of such results, a \%RM value was used for the training/research protocols with different tempos despite the $1 \mathrm{RM}$ values were likely different for each tempo, as this occurred in the current investigation. Given that a slow or an extremely slow tempo of movement causes a decrease in the maximal load during the $1 \mathrm{RM}$ test above $10 \%$, the value of \%1RM should also be determined upon such a result. Therefore, when different movement tempos are used in testing protocols, $1 \mathrm{RM}$ performance should be assessed for each tempo.

Although the present study provides valuable information in terms of the effect of extending the ECC phase on the 1RM load, this study only investigated the BP exercise. Therefore, it is possible that other exercises may have different limiting factors during 1RM testing and that the findings presented here may not apply to other exercises. Furthermore, there was no direct analysis of physiological variables which could explain the results of the present study. Since there is no evidence regarding the possible cause for changes in the maximal load, further research is needed to assess physiological and biochemical variables describing acute changes following resistance exercises with different movement tempos.

\section{Conclusions}

The movement tempo has a significant impact on the load lifted in a 1RM test. An increase in the duration of the ECC phase proportionally decreases the maximal load lifted during the 1RM test. The results of this study indicate that during research or training, when a controlled movement tempo is used, 1RM testing should be performed independently for particular movement tempos. The \%1RM value should be determined upon the 1RM test trial at a specific movement tempo. In addition, the standardization of the tempo should be taken into account in investigations that use $1 \mathrm{RM}$ tests to assess the effects on any treatment involving maximal muscle strength.

\section{Acknowledgements}

This study would not have been possible without our participants' commitment, time and effort. The study was supported and funded by the statutory research of the Jerzy Kukuczka Academy of Physical Education in Katowice, Poland 


\section{References}

Cohen J. Statistical Power Analysis for the Behavioral Sciences. 2nd ed. Routledge; 2013. doi:10.4324/9780203771587

Cronin JB, Henderson ME. Maximal strength and power assessment in novice weight trainers. J Strength Cond Res, 2004; 18(1): 48-52. doi:10.1519/00124278-200402000-00006

Cronin JB, McNair PJ, Marshall RN. Magnitude and decay of stretch-induced enhancement of power output. Eur J Appl Physiol, 2001; 84(6): 575-81. doi:10.1007/s004210100433

Goto K, Ishii N, Kizuka T, Kraemer RR, Honda Y, Takamatsu K. Hormonal and metabolic responses to slow movement resistance exercise with different durations of concentric and eccentric actions. Eur J Appl Physiol, 2009; 106(5): 731-9. doi:10.1007/s00421-009-1075-9

Headley SA, Henry K, Nindl BC, Thompson BA, Kraemer WJ, Jones MT. Effects of Lifting Tempo on One Repetition Maximum and Hormonal Responses to a Bench Press Protocol. J Strength Cond Res, 2011; 25(2): 406-13. doi:10.1519/JSC.0b013e3181bf053b

Kraemer WJ, Ratamess NA, Fry AC, French DN. Strength training: development and evaluation of methodology. In: Physiological assessment of human fitness. Maud P.J., Foster C, editors. Champain IL: Human Kinetics; 2006

Levinger I, Goodman C, Hare DL, Jerums G, Toia D, Selig S. The reliability of the 1RM strength test for untrained middle-aged individuals. J Sci Med Sport, 2009; 12(2): 310-6. doi:10.1016/j.jsams.2007.10.007

Martins-Costa HC, Diniz RCR, Lima FV, Machado SC, Almeida RS, Andrade AG, Chagas MH. Longer repetition duration increases muscle activation and blood lactate response in matched resistance training protocols. Motriz Revista de Educação Física, 2016; 22(1): 35-41. doi:10.1590/S198065742016000100005

Mazzetti S, Douglass M, Yocum A, Harber M. Effect of Explosive versus Slow Contractions and Exercise Intensity on Energy Expenditure. Med Sci Sports Exerc, 2007; 39(8): 1291-301. doi:10.1249/mss.0b013e318058a603

Maszczyk A, Dobrakowski P, Żak M, Gozdowski P, Krawczyk M, Małecki A, Stastny P, Zajac T. Differences in motivation during the bench press movement with progressive loads using EEG analysis. Biol Sport, 2019; 36(4): 351-6. https://doi.org/10.5114/biolsport.2019.88757

Maszczyk A, Wilk M, Krzysztofik M, Gepfert M, Zając A, Petr M, Stastny P. The effects of resistance training experience on movement characteristics in the bench press exercise. Biology of Sport, 2020; 37(1): 79-83. doi:10.5114/biolsport.2019.83008

Mike JN, Cole N, Herrera C, VanDusseldorp T, Kravitz L, Kerksick CM. The Effects of Eccentric Contraction Duration on Muscle Strength, Power Production, Vertical Jump, and Soreness. J Strength Cond Res, 2017; 31(3): 773-86. doi:10.1519/JSC.0000000000001675

Miller RM, Freitas ED, Heishman AD, Kaur J, Koziol KJ, Galletti BA, Bemben NG. Maximal power production as a function of sex and training status. Biol Sport, 2018; 36(1): 31-7. doi: 10.5114/biolsport.2018.78904

Newton RU, Murphy AJ, Humphries BJ, Wilson GJ, Kraemer WJ, Häkkinen K. Influence of load and stretch shortening cycle on the kinematics, kinetics and muscle activation that occurs during explosive upperbody movements. Eur J Appl Physiol, 1997; 75(4): 333-42. doi:10.1007/s004210050169

Schoenfeld BJ, Ogborn DI, Krieger JW. Effect of Repetition Duration During Resistance Training on Muscle Hypertrophy: A Systematic Review and Meta-Analysis. Sports Med, 2015; 45(4): 577-85. doi:10.1007/s40279-015-0304-0

Seo DI, Kim E, Fahs CA, Rossow L, Young K, Ferguson SL, Thiebaud R, Sherk VD, Loenneke JP, Kim D, Lee MK, Choi KH, Bemben DA, Bemben MG, So WY. Reliability of the one-repetition maximum test based on muscle group and gender. J Sports Sci Med, 2012; 11(2): 221-5 
Shimano T, Kraemer WJ, Spiering BA, Volek JS, Hatfield DL, Silvestre R, Vingren JL, Fragala MS, Maresh CM, Fleck SJ, Newton RU, Spreuwenberg LP, Häkkinen K. Relationship Between the Number of Repetitions and Selected Percentages of One Repetition Maximum in Free Weight Exercises in Trained and Untrained Men. J Strength Cond Res, 2006; 20(4): 819. doi:10.1519/R-18195.1

Wilk M, Golas A, Krzysztofik M, Nawrocka M, Zajac A. The Effects of Eccentric Cadence on Power and Velocity of the Bar during the Concentric Phase of the Bench Press Movement. J Sports Sci Med, 2019; 18(2): 191-7

Wilk M, Golas A, Stastny P, Nawrocka M, Krzysztofik M, Zajac A. Does Tempo of Resistance Exercise Impact Training Volume? J Hum Kinet, 2018a; 62(1): 241-50. doi:10.2478/hukin-2018-0034

Wilk M, Stastny P, Golas A, Nawrocka M, Jelen K, Zajac A, Tufano JJ. Physiological responses to different neuromuscular movement task during eccentric bench press. Neuro Endocrinol Lett, 2018b; 39(1): 26-32

Wilk M, Gepfert M, Krzysztofik M, Mostowik A, Filip A, Hajduk G, Zajac A. Impact of duration of eccentric movement in the one-repetition maximum test result in the bench press among women. J Sports Sci Med, 2020; 19: 317-22

\section{Corresponding author:}

\section{dr Michal Wilk}

Institute of Sport Sciences,

Jerzy Kukuczka Academy of Physical Education in Katowice,

Poland

E-mail: m.wilk@awf.katowice.pl 